KUNS-1598

hep-th/9909027

\title{
Corrections to D-brane Action and Generalized Boundary State
}

\author{
Koji Hashimoto* \\ Department of Physics, Kyoto University, Kyoto 606-8502, Japan
}

September, 1999

\begin{abstract}
In this paper, we generalize a boundary state to the one incorporating nonconstant gauge field strength as an external background coupled to the boundary of a string worldsheet in bosonic string theory. This newly defined boundary state satisfies generalized nonlinear boundary conditions with non-constant gauge field strength, and is BRST invariant. The divergence immanent in this boundary state coincide with the one calculated in a string $\sigma$ model. We extract the relevant massless part of this generalized boundary state, and give a part of the D-brane action with the non-constant gauge field strength, that is, derivative corrections to the D-brane action.
\end{abstract}

*hasshan@gauge.scphys.kyoto-u.ac.jp 


\section{Introduction}

D-branes [1] are indispensable objects in string theories to the understanding of their nonperturbative aspects, including various dualities [2]. One way to examine string dualities is to use the D-brane action which is a low energy effective action of massless fields induced on the D-brane [3]. For example, taking the worldvolume dual of the D1-brane action, we obtain a Nambu-Goto string action with a tension rotated by $S L(2, Z)$ duality transformation which is conjectured to exist in type IIB superstring theory [4]. Similar analyses have been performed for other cases, such as D $p$-brane $(2 \leq p \leq 4)$ actions or super- $p$-brane actions [4. 5]. As another argument on string dualities using D-brane action we cite ref. [6], which shows that the BPS mass spectra given as a volume factor of the D-brane action describing compactified D-branes are consistent with U-duality.

The D-brane actions used in the above analyses are the ones given by the lowest order calculation in the string $\sigma$ model approach [7, 8, 9, 10], or in the open string partition function approach [11], in addition to the ordinary scattering amplitude approach [12]. Higher order calculations of the D-brane actions have been known through the latter approaches [13], in the trivial background of the massless sector of the closed string excitations (flat metric and no Kalb-Ramond two-form field). This adds to the D-brane action higher derivative $\left(\alpha^{\prime}\right)$ corrections. . systematic framework which gives higher derivative corrections to the D-brane action is highly desired.

Since D-branes are defined as hypersurfaces on which the boundaries of the string worldsheets can sit, the boundary states [17, 18, 19, 20, 21] describe the dynamics of the D-branes well. A part of the D-brane action (more precisely, couplings to the fields of the closed string excitations) is encoded in the boundary state [22]. However, this boundary state is defined as an eigen state of the boundary conditions for the oscillating modes of the attached string, the explicit form of the boundary state has been given only for simple backgrounds which give linear boundary conditions. One of those configurations is the constant field strength on the D-brane. Therefore, boundary states including general configuration of the boundary gauge field give us a method to calculate the higher derivative corrections to the D-brane action.

In ref. [23], a D-brane sector was introduced to the system of covariant closed bosonic

\footnotetext{
${ }^{1}$ There are some arguments that the supersymmetry ensures the absence of correction terms to the D-brane action [14].

${ }^{2}$ T-duality for the bulk supergravity action can be extended to the one including the higher loop effects (see ref. [15]). In other references, the higher derivative corrections to the D-brane action in the gravity sector (especially, (curvature) $)^{2}$ terms) are calculated and its consistency with string dualities is checked [16].
} 
string field theory (SFT) [24]. The D-brane action corresponds to the source term in this SFT, and the boundary state is a constituent of it. The dynamical degree of freedom of the boundary state adopted there was only the constant field strength. In this scheme of SFT, a $\sigma$ model gauge transformation was realized is such a way that it yields a constant shift of the gauge field strength in the boundary state.

In this paper, we present a generalized boundary state, which is constructed by a gauge transformation in the SFT. This boundary state incorporates the non-constant modes of $F_{\mu \nu}(x)$ and gives the derivative corrections to the D-brane actions. Our new boundary state is defined for an arbitrary gauge field configuration $A_{\mu}(x)$, and we carry out the explicit calculations up to the second order in the derivatives on $F_{\mu \nu}(x)$. This boundary state includes divergences, and the requirement for these divergences to vanish gives a constraint on the configuration of the gauge field $A_{\mu}(x)$. This constraint is shown to be identical with the vanishing of the $\beta$ function in a string $\sigma$ model, hence we regard this constraint as an equation of motion for the gauge field. After this regularization procedure for the boundary state, we obtain the higher derivative corrections to the D-brane action, by extracting relevant parts in the boundary state. It is expected that this generalized boundary state serves as an useful tool for the investigation of the dualities or other dynamics in string theory.

The organization of the rest of this paper is as follows. In the next section, after summarizing the properties of the boundary states adopted in ref. [23], this boundary state is generalized to the one which has non-constant $F_{\mu \nu}(x)$ boundary coupling. In sec. 3, we study the divergence immanent in the generalized boundary state, and see the coincidence with a string $\sigma$ model. In sec. 1 , using the new boundary state proposed in sec. 2, we calculate the correction to the D-brane action. The final section is devoted to our conclusion and discussions. We include an appendix where the calculation of the formula appearing in this paper is given.

\section{Generalizing the boundary state}

As mentioned in the introduction, a certain sector of the boundary state is related to the low energy effective action of D-branes [22]. The boundary state adopted in ref. [23] and in most of the literatures has a single free parameter, that is, constant field strength of the gauge field

\footnotetext{
${ }^{3}$ We concentrate on the bosonic string theory in this paper. A work on supersymmetric extension will appear soon 25].
} 
on the D-brane.f Therefore the obtained effective action on the D-brane contains only the constant field strength. In this section, we generalize the boundary state so that it includes non-constant modes of the field strength, in order to calculate the corrections to the D-brane action. These corrections correspond to the derivative corrections, since we expand the field strength by derivatives.

First let us summarize the relevant properties of the boundary state with the constant field strength. The boundary state $|B(F)\rangle$ is defined as an eigen state of the boundary conditions for open bosonic strings [17, 18, 20]:

$$
\begin{aligned}
& X^{i}(\sigma)|B(F)\rangle=0 \\
& \left(\pi P_{\mu}(\sigma)+F_{\mu \nu} \partial_{\sigma} X^{\nu}(\sigma)\right)|B(F)\rangle=0, \\
& \pi_{c}(\sigma)|B(F)\rangle=\pi_{\bar{c}}(\sigma)|B(F)\rangle=0 .
\end{aligned}
$$

The BRST invariance of the boundary state is a consequence of eqs. (2.1) - (2.3). The oscillator representation of $|B(F)\rangle$ reads

$$
\left|B(F)\left(x^{M}, \bar{c}_{0}, \widetilde{\alpha}\right)\right\rangle=N(F)\left|B_{\mathrm{N}}(F)\right\rangle \otimes\left|B_{\mathrm{D}}\right\rangle \otimes\left|B_{\mathrm{gh}}\right\rangle
$$

where

$$
\begin{aligned}
& \left|B_{\mathrm{N}}(F)\right\rangle=\exp \left\{-\sum_{n \geq 1} \frac{1}{n} \alpha_{-n}^{(-) \mu} \mathcal{O}(F)_{\mu}{ }^{\nu} \alpha_{-n \nu}^{(+)}\right\}|0\rangle_{p+1} \\
& \left|B_{\mathrm{D}}\right\rangle=\exp \left\{\sum_{n \geq 1} \frac{1}{n} \alpha_{-n}^{(-) i} \alpha_{-n i}^{(+)}\right\}|0\rangle_{d-p-1} \delta^{d-p-1}\left(x^{i}\right) \\
& \left|B_{\mathrm{gh}}\right\rangle=\exp \left\{\sum_{n \geq 1}\left(c_{-n}^{(-)} \bar{c}_{-n}^{(+)}+c_{-n}^{(+)} \bar{c}_{-n}^{(-)}\right)\right\}|0\rangle_{\mathrm{gh}} .
\end{aligned}
$$

The orthogonal matrix $\mathcal{O}$ is defined as $\mathcal{O}_{\mu}{ }^{\nu}=(1-F)_{\mu}{ }^{\rho}\left\{(1+F)^{-1}\right\}_{\rho}{ }^{\nu}$. Although the front factor $N(F)$ is arbitrary, this can be fixed by various ways [22, 23, 26]. Here we shall follow ref. [23], where the gauge invariance principle for the D-brane (source) term in the closed SFT action has fixed the front factor. Only when the front factor is given by

$$
N(F)=-\frac{T_{p}}{4}(\operatorname{det}(1+F))^{-\zeta(0)}
$$

the boundary state is subject to the following SFT gauge transformation:

$$
|\Lambda * B(F)\rangle=\delta_{\Lambda}|B(F)\rangle
$$

\footnotetext{
${ }^{4}$ D-branes moving with a constant velocity or tilted by a constant angle are treated as a T-dual (or boosted) version of the case with the constant field strength [26, 27].
} 
where the $*$ product denotes three string interaction vertex. A string field $\Lambda$ is a gauge transformation parameter

$$
\left|\Lambda\left(\zeta_{\mu}(x)\right)\right\rangle=-i \bar{c}_{0} \zeta_{\mu}(x)\left(\bar{c}_{-1}^{(+)} \alpha_{-1}^{(-) \mu}-\bar{c}_{-1}^{(-)} \alpha_{-1}^{(+) \mu}\right)|0\rangle
$$

with

$$
\zeta_{\mu}(x)=\zeta_{\mu \nu} x^{\nu}
$$

and we have defined the gauge transformation for the variable $F$ as

$$
\delta_{\Lambda} F_{\mu \nu}=\zeta_{\mu \nu}-\zeta_{\nu \mu}
$$

For verifying the gauge transformation (2.9), we have used a relation on the $*$ product

$$
|\Lambda * B(F)\rangle=-\frac{i}{2 \pi} \oint d \sigma \partial_{\sigma} X^{\mu} \zeta_{\mu \nu} X^{\nu}|B(F)\rangle,
$$

for the above $\Lambda$ (2.10).

The gauge transformation for the closed string field is given by

$$
\delta_{\Lambda} \Phi=Q_{\mathrm{B}} \Lambda+2 \Phi * \Lambda
$$

therefore with the transformation parameter (2.10), the inhomogeneous part of the gauge transformation (2.14) generates a shift of a component Kalb-Ramond gauge field $b_{\mu \nu}(x)$ in $\Phi$ by $\partial_{\mu} \zeta_{\nu}(x)-\partial_{\nu} \zeta_{\mu}(x)$. (Our notation of SFT is given in ref. [23.) This fact is expected from the gauge symmetry of the string $\sigma$ model, which is defined as $\delta_{(\sigma \text { model })} A_{\mu}(x)=-\zeta_{\mu}(x)$ and $\delta_{(\sigma \text { model })} b_{\mu \nu}(x)=\partial_{\mu} \zeta_{\nu}(x)-\partial_{\nu} \zeta_{\mu}(x)$ 9]. Note that in the string $\sigma$ model approach, this gauge transformation $\delta_{(\sigma \text { model })}$ generates all the modes of the gauge field $A_{\mu}(x)$ on the boundary. Therefore, in order to generalize the boundary state to the one incorporating the functional degree of freedom $A_{\mu}(x)$ (or $F_{\mu \nu}(x)$ ), it is natural to demand the following relation for the new boundary state $\left|\mathcal{B}\left(A_{\mu}(x)\right)\right\rangle$ :

$$
\delta_{\Lambda}|\mathcal{B}\rangle=-2|\Lambda * \mathcal{B}\rangle
$$

In this relation the parameter string field $\Lambda$ is defined by (2.10) for an arbitrary function $\zeta_{\mu}(x)$, and the gauge transformation law concerning $A_{\mu}(x)$ is

$$
\delta_{\Lambda} A_{\mu}(x)=-\zeta_{\mu}(x)
$$

Since the gauge transformation (2.16) generates an arbitrary deformation of $A_{\mu}(x)$ by the parameter $\zeta_{\mu}(x),\left|\mathcal{B}\left(A_{\mu}(x)\right)\right\rangle$ is obtained by "integrating" eq. (2.15). For this purpose we use 
the following formula for the star product $\Lambda\left(\zeta_{\mu}(x)\right) * \Psi$ between the present $\Lambda$ and an arbitrary string functional $\Psi$ (the derivation is given in app. A):

$$
-2\left|\Lambda\left(\zeta_{\mu}(x)\right) * \Psi\right\rangle=\left\{\frac{i}{\pi} \int_{0}^{2 \pi} d \sigma \partial_{\sigma} X^{\mu} \zeta_{\mu}(X)+\left.\left.\int_{0}^{2 \pi} d \sigma \partial^{\mu} \zeta_{\mu}(X) i \pi_{c}\right|_{\text {oscil. }} i \pi_{\bar{c}}\right|_{\text {oscil. }}\right\}|\Psi\rangle \text {. }
$$

This is a generalization of the identity (2.13). Assuming that the ghost part of the new boundary state is given by the same form (eq. (2.7)) as before, the second term on the RHS of eq. (2.17) acting on the state $|\mathcal{B}\rangle$ vanishes due to the ghost boundary condition (2.3). Then the desired generalized boundary state $|\mathcal{B}\rangle$ is obtained by exponentiating the operator on the RHS of (2.17): :

$$
\left|\mathcal{B}\left(A_{\mu}(x)\right)\right\rangle \equiv U[A]|B(F=0)\rangle
$$

with

$$
U[A] \equiv \exp \left(\frac{-i}{\pi} \oint d \sigma \partial_{\sigma} X^{\mu} A_{\mu}(X)\right)
$$

Note that $U[A]$ and hence $\mathcal{B}$ has an invariance under the $U(1)$ gauge transformation of $A_{\mu}(x)$, $A_{\mu} \rightarrow A_{\mu}+\partial_{\mu} \epsilon$.

The new boundary state (2.18) is reduced to the previous one (2.4) when the dynamical variable is restricted to the constant field strength

$$
A_{\mu}(x) \equiv a_{\mu \nu}^{(1)} x^{\nu}, \quad-\left(a_{\mu \nu}^{(1)}-a_{\nu \mu}^{(1)}\right)=F_{\mu \nu}
$$

since the Neumann part of the previous boundary state (2.4) is written in a form

$$
\begin{aligned}
N(F)\left|B_{\mathrm{N}}(F)\right\rangle & =\exp \left(\frac{i}{2 \pi} \oint d \sigma \partial_{\sigma} X^{\mu} F_{\mu \nu} X^{\nu}\right)\left|B_{\mathrm{N}}(F=0)\right\rangle \\
& =\exp \left(\frac{-i}{\pi} \oint d \sigma \partial_{\sigma} X^{\mu} A_{\mu}(X)\right)\left|B_{\mathrm{N}}(F=0)\right\rangle .
\end{aligned}
$$

Our $|\mathcal{B}\rangle$ satisfies various desired properties. One of them is the fact that it obeys the following generalized nonlinear boundary condition

$$
\left[\pi P_{\mu}+F_{\mu \nu}(X) \partial_{\sigma} X^{\nu}\right]|\mathcal{B}\rangle=0
$$

where the field strength $F_{\mu \nu}(X)$ is defined as usual:

$$
F_{\mu \nu}(X)=\partial_{\mu} A_{\nu}(X)-\partial_{\nu} A_{\mu}(X) .
$$

\footnotetext{
5 This definition is very natural in the sense of path-integral approach of string $\sigma$ model [18]. The boundary state is regarded as an initial or final wave functional for the path-integral. The rotational operator $U[A]$ is a path-integral weight $\exp \left(i S_{\text {int }}^{(\sigma)}\right)$, where $S_{\text {int }}^{(\sigma)}$ is a minimal interaction term with external gauge fields $A_{\mu}(X)$ in the string $\sigma$ model action.
} 
This eigen equation (2.23) is easily proved from a property of the unitary operator $U[A](2.19)$ :

$$
U[A] \pi P_{\mu} U[A]^{-1}=\pi P_{\mu}+F_{\mu \nu}(X) \partial_{\sigma} X^{\nu}
$$

and $P_{\mu}|B(F=0)\rangle=0$. The new boundary state $|\mathcal{B}\rangle$ satisfies a generalized boundary condition (2.23) which is in the same form required in the scheme of the string $\sigma$ model [8, 9, 18]. Thus also from this point of view it is possible to assert that this state $|\mathcal{B}\rangle$ really describes a boundary of a string worldsheet coupled to a massless gauge field $A_{\mu}(x)$.

One of the other properties of $|\mathcal{B}\rangle$ is the BRST invariance which is important to understand loop-corrected equations of motion for closed string theory [18, [19] . The BRST charge can be written in the form (see Appendix A in the ref. [23] for its complete expression)

$$
Q_{\mathrm{B}}=2 \sqrt{\pi} \oint d \sigma\left\{i \pi_{\bar{c}} \cdot\left[\text { functions of } P_{M} \text { and } X^{N}\right]-c P_{M} \partial_{\sigma} X^{M}\right\}+Q_{\mathrm{B}}^{\text {(ghost) }},
$$

where the term $Q_{\mathrm{B}}^{\text {(ghost) }}$ consists only of the ghost coordinates. Noting that the ghost part of the new boundary state $|\mathcal{B}\rangle_{\text {ghost }}$ is the same as the previous one $\left|B_{\text {gh }}\right\rangle$, we have

$$
\pi_{\bar{c}}|\mathcal{B}\rangle=Q_{\mathrm{B}}^{\text {(ghost) }}|\mathcal{B}\rangle=0 .
$$

Then the commutativity

$$
\left[P_{\mu} \partial_{\sigma} X^{\mu}, \oint d \sigma^{\prime} \partial_{\sigma^{\prime}} X^{\nu} A_{\nu}(X)\right]=0
$$

ensures the BRST invariance

$$
Q_{\mathrm{B}}|\mathcal{B}\rangle=0
$$

Although this boundary state $|\mathcal{B}\rangle$ is a solution of the equation (2.29) for arbitrary configuration of the gauge field, this solution is formal, in a sense that the boundary state contains divergence and thus is not well-defined unless regularized. In the next section we study on this point in detail.

\footnotetext{
${ }^{6}$ In the closed SFT, the D-brane action as well as the usual closed string field action is implemented [23] as $S=\frac{1}{2}\left\langle\Phi\left|Q_{\mathrm{B}}\right| \Phi\right\rangle+\langle\Phi \mid B\rangle$, where we consider only the linearized part. The equation of motion follows as $Q_{\mathrm{B}}|\Phi\rangle+|B\rangle=0$, hence multiplying $Q_{\mathrm{B}}$ on this expression we obtain a constraint $Q_{\mathrm{B}}|B\rangle=0$. This constraint of the BRST invariance of the boundary state is also deduced from the gauge invariance of the whole SFT action 23.
} 


\section{Divergence in the new boundary state}

\subsection{Calculation of the divergence}

The new boundary state (2.18) for a general (polynomial) $A_{\mu}(x)$ is made of products of the operators $X$ 's at the same point $\sigma$ and therefore it inevitably contains divergences. We shall show in this subsection that these divergences can be absorbed into the redefinition of the gauge field as $A_{\mu}^{\text {red }}(x) \equiv A_{\mu}(x)+A_{\text {divergent. }}$. We require that the divergent part $A_{\text {divergent }}$ to vanish, in order to obtain a well-defined boundary state. Eventually, constraint equation is found to be identical with the vanishing of the $\beta$ function of the string $\sigma$ model coupling. Thus this requirement of no-divergence coincides with the condition of the conformal invariance in the string $\sigma$ model.

For studying the divergences in the new boundary state, we divide $X^{\mu}(\sigma)$ into the zero mode and the non-zero mode part $\widetilde{X}^{\mu}(\sigma)$,

$$
X^{\mu}(\sigma)=x^{\mu}+\widetilde{X}^{\mu}(\sigma),
$$

and Taylor-expand $A_{\mu}(X(\sigma))$ around the zero mode $x^{\mu}$ as

$$
A_{\mu}(X)=A_{\mu}(x)+\widetilde{X}^{\nu} \partial_{\nu} A_{\mu}(x)+\frac{1}{2} \widetilde{X}^{\nu} \widetilde{X}^{\rho} \partial_{\nu} \partial_{\rho} A_{\mu}(x)+\cdots .
$$

We denote the contribution of the second term on the RHS of (3.2) to $U[A](2.19)$ by $U_{0}$ and that of the rest terms by $V$, and express $U[A]$ as

$$
U[A]=V U_{0} .
$$

Explicitly, we have

$$
U_{0}=\exp \left(\frac{i}{2 \pi} \oint d \sigma \partial_{\sigma} X^{\mu} X^{\nu} F_{\mu \nu}(x)\right)
$$

and

$$
\begin{array}{r}
V=1+\frac{-i}{3 \pi} \oint d \sigma \partial_{\sigma} \widetilde{X}^{\mu} \widetilde{X}^{\nu} \widetilde{X}^{\rho} \partial_{\rho} F_{\nu \mu}(x)+\frac{-i}{8 \pi} \oint d \sigma \partial_{\sigma} \widetilde{X}^{\mu} \widetilde{X}^{\nu} \widetilde{X}^{\rho} \widetilde{X}^{\delta} \partial_{\delta} \partial_{\rho} F_{\nu \mu}(x) \\
+\frac{1}{2}\left(\frac{-i}{3 \pi} \oint d \sigma \partial_{\sigma} \widetilde{X}^{\mu} \widetilde{X}^{\nu} \widetilde{X}^{\rho} \partial_{\rho} F_{\nu \mu}(x)\right)^{2}+\cdots
\end{array}
$$

In (3.5) we have written explicitly only those terms where the total number of derivatives acting on $F_{\mu \nu}(x)$ is at most two. 
Since the operator $U_{0}$ acting on $|B(F=0)\rangle$ generates the "old" boundary state (2.4) with $x$-dependent field strength (cf., eq. (2.21)),

$$
U_{0}|B(F=0)\rangle=|B(F(x))\rangle
$$

the new boundary state $(2.18)$ is expressed as

$$
|\mathcal{B}\rangle=V|B(F(x))\rangle .
$$

Note that the approximation to the first order with respect to the derivative acting on the field strength, where $V$ is put to be 1 , does not reduce $|\mathcal{B}\rangle$ to the previous (BRST invariant) boundary state $|B(F)\rangle$ considered in the ref. [23]. Actually, the decomposition of $U[A]$ into $U_{0} V$ does not respect the BRST invariance at each order in the expansion of $V$.

Next, we shall evaluate the representation (3.7) by expressing it only by the creation operators acting upon $|B(F(x))\rangle$. Namely, we change all the annihilation operators in $V$ into the creation ones with use of the Neumann boundary condition satisfied by $|B(F(x))\rangle$ :

$$
\left(\alpha_{n \mu}^{(-)}+\mathcal{O}_{\mu}^{\nu}(x) \alpha_{-n \nu}^{(+)}\right)|B(F(x))\rangle=0
$$

where $\mathcal{O}(x)$ is an orthogonal matrix defined by

$$
\mathcal{O}_{\mu}{ }^{\nu}(x) \equiv\left(\frac{1-F(x)}{1+F(x)}\right)_{\mu}^{\nu}, \quad \mathcal{O O}^{T}=\mathcal{O}^{T} \mathcal{O}=1
$$

The calculation is straightforward and we here present only the results (see app. B for the detailed calculation). The unitary operator $V$ reexpressed in terms of creation operators contains two kinds of divergences,

$$
\sum_{n=1}^{\infty} 1=\zeta(0), \sum_{n=1}^{\infty} \frac{1}{n}=\zeta(1) .
$$

Adopting the zeta function regularization, $\zeta(0)=-1 / 2$, we find that the $\zeta(1)$ divergence is absorbed into the redefinition of the field strength $F(x)$ :

$$
|\mathcal{B}\rangle=\left|B\left(F^{\mathrm{red}}(x)\right)\right\rangle+\left[\begin{array}{l}
\text { creation operators } \\
\text { with finite coefficients }
\end{array}\right]|B(F(x))\rangle
$$

where the redefined $F^{\text {red }}(x)$ containing the $\zeta(1)$ divergence is given by

$$
\begin{aligned}
F_{\mu \nu}^{\mathrm{red}}(x) \equiv F_{\mu \nu}(x)+ & \frac{1}{2} \zeta(1)\left(\frac{1}{1+F}\right)^{\rho \delta} \partial_{\rho} \partial_{\delta} F_{\mu \nu} \\
+ & \frac{1}{4} \zeta(1)\left[\left\{\left(\frac{1}{1+F}\right)^{\lambda \rho} \partial_{\nu} F_{\rho \delta}\left(\frac{1}{1+F}\right)^{\delta \kappa}\left(\partial_{\lambda} F_{\kappa \mu}+\partial_{\kappa} F_{\lambda \mu}\right)\right\}-\{\mu \leftrightarrow \nu\}\right] .
\end{aligned}
$$


Here we have kept only those terms written explicitly in eq. (3.5).1

The redefinition (3.12) can be expressed in terms of gauge field $A_{\mu}$ as

$$
A_{\mu}^{\mathrm{red}}(x) \equiv A_{\mu}(x)+\frac{1}{2} \zeta(1)\left(\frac{1}{1-F(x)^{2}}\right)^{\lambda \nu} \partial_{\lambda} F_{\nu \mu}(x) .
$$

For the generalized boundary state to be well-defined (not divergent), the coefficient of the $\zeta(1)$ in eq. (3.13) should put equal to zero. Therefore we obtain a constraint on the configuration of the gauge field

$$
\beta(A) \equiv\left(\frac{1}{1-F(x)^{2}}\right)^{\lambda \nu} \partial_{\lambda} F_{\nu \mu}(x)=0 .
$$

At a glance one recognizes that this $\beta(A)$ is identical with the beta function for the gauge field coupling in the string $\sigma$ model [8]. Hence the constraint (3.14) for the generalized boundary state coincides with the conformal invariance condition in the string $\sigma$ model. We shall explain the detailed correspondence between our formalism and the string $\sigma$ model approach in the next subsection.

\subsection{Correspondence to the string $\sigma$ model $\beta$ function}

We have seen in the previous subsection that the divergences immanent in the newly defined boundary state coincide with the result in the string $\sigma$ model. This feature is natural in a sense that, in the both formalisms, one evaluates the same weight $\exp \left(S_{\text {int }}^{(\sigma)}\right)$ (see the footnote 5) on the boundary of the string worldsheet. However, two approaches appear to be clearly different in the following respect: although the $\sigma$ model approach gives a loop contribution to the gauge potential itself, the divergences in the boundary state show up in the form of the redefinition of the field strength. In this subsection we study how the divergences in the boundary state can be understood in the scheme of the string $\sigma$ model.

Consider a string $\sigma$ model action [7, 8, 9]

$$
S=\frac{1}{\pi}\left[\frac{1}{2} \int d^{2} \sigma \partial_{\alpha} X_{M} \partial^{\alpha} X^{M}+\oint d \sigma\left(X^{\mu}\right)^{\prime} A_{\mu}[X]\right]
$$

where the prime denotes the derivative with respect to a worldsheet coordinate $\sigma$. The boundary of the worldsheet is put at $\tau=0$, since we intend to study the correspondence with the

7 The first derivative correction to the boundary state (or the Born-Infeld action) should contain two derivatives acting on $F$, since if the corrected action is written by gauge invariant field strength $F$, the number of indices should be even. 
boundary state and therefore treat a closed string emitted by the D-brane. Expand the action (3.15) around a classical configuration $\bar{X}$ as

$$
\begin{aligned}
i \pi S[\bar{X}+\xi]= & {\left[\frac{1}{2} \int d^{2} \sigma \partial_{\alpha} \bar{X}_{M} \partial^{\alpha} \bar{X}^{M}-i \oint d \sigma\left(\bar{X}^{\mu}\right)^{\prime} A_{\mu}[\bar{X}]\right] } \\
& +\left[\int d^{2} \sigma \partial_{\alpha} \bar{X}_{M} \partial^{\alpha} \xi^{M}-i \oint d \sigma \xi^{\mu}\left(\bar{X}^{\nu}\right)^{\prime} F_{\mu \nu}[\bar{X}]\right] \\
& +\left[\frac{1}{2} \int d^{2} \sigma \partial_{\alpha} \xi_{M} \partial^{\alpha} \xi^{M}-\frac{i}{2} \oint d \sigma \xi^{\mu}\left(\xi^{\nu}\right)^{\prime} F_{\mu \nu}[\bar{X}]\right] \\
& -i \oint d \sigma\left(\frac{1}{2} \xi^{\rho} \xi^{\nu}\left(\bar{X}^{\mu}\right)^{\prime} \partial_{\nu} F_{\rho \mu}[\bar{X}]\right. \\
& \left.\quad+\frac{1}{3} \xi^{\nu} \xi^{\rho}\left(\xi^{\mu}\right)^{\prime} \partial_{\nu} F_{\rho \mu}[\bar{X}]+\frac{1}{8} \xi^{\delta} \xi^{\rho} \xi^{\nu}\left(\xi^{\mu}\right)^{\prime} \partial_{\delta} \partial_{\rho} F_{\nu \mu}[\bar{X}]+\cdots\right)
\end{aligned}
$$

where the fluctuation around the classical configuration is denoted as $\xi$ and we have adopted Euclidean formalism, $i \tau \rightarrow \tau$. In this expansion, we are working in slowly varying fields, i.e. derivative expansion.

In the above equation (3.16), terms in the second line vanishes due to the equations of motion for the classical configuration $\bar{X}$. The terms in the third line gives a kinetic term (propagator) for the scalar field $\xi$. This propagator depends on the constant field strength $F$ on the boundary. Usually in the string $\sigma$ model calculation [7, 8], one evaluates a vacuum graph using the vertex in the fourth line by the contraction

$$
\oint d \sigma \prod_{\xi^{\rho} \xi^{\nu}}\left(\bar{X}^{\mu}\right)^{\prime} \partial_{\nu} F_{\rho \mu}[\bar{X}]
$$

This quantity is in proportion to $(\bar{X})^{\prime}$, therefore the divergence of this one-loop vacuum graph is interpreted as the one-loop divergent quantum contribution to the gauge field $A_{\mu}[X]$ in the coupling $\oint d \sigma\left(\bar{X}^{\mu}\right)^{\prime} A_{\mu}[\bar{X}]$ which appears in the first line in eq. (3.16).

However, when we ignore the first line and only consider the dynamics of the scalar field $\xi(\sigma, \tau)$, it is natural to calculate the renormalization of the propagator, three point vertex, and so on. Let us concentrate on the one-loop correction to the propagator. Using the couplings in the fifth line in eq. (3.16), at one-loop level we have two relevant Feynman graphs shown in Fig. 1 and 2, which bring out divergences in the sector of the propagator. Though generally the divergence in the two-point function is absorbed into the renormalization factor of the wave function, now according to the philosophy of the string $\sigma$ model, we expect that these divergences can be absorbed into the redefinition of the gauge field strength in the propagator, with the form (3.12).

We demonstrate here the calculation of the $\partial \partial F$ divergences depicted in Fig. 1. With use 


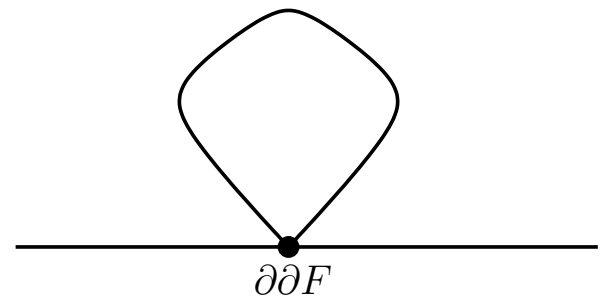

Figure 1: Contribution of the $\partial \partial F$ term to the propagator with one-loop. The solid lines are propagation of the fluctuation $\xi$, and the interaction vertex $\partial \partial F$ is on the boundary.

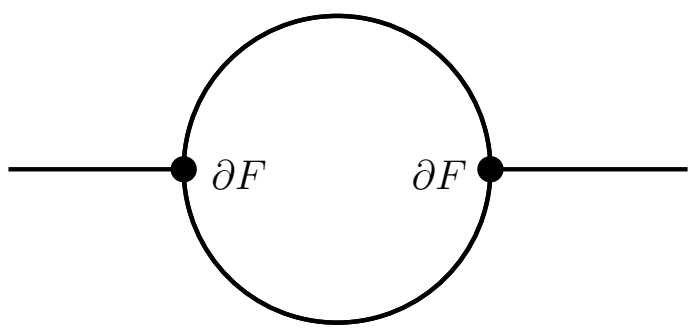

Figure 2: Contribution of the $(\partial F)^{2}$ term to the propagator with one-loop.

of the oscillator representation of the boundary condition for the constant field strength (2.2),

$$
\alpha_{n \mu}^{(-)}=-\mathcal{O}_{\mu}^{\nu} \alpha_{-n \nu}^{(+)}
$$

we obtain an explicit form of $X$ on the boundary as

$$
X_{\mu}=x_{\mu}+\frac{i}{2} \sum_{n \neq 0} \alpha_{n \nu}^{(+)}\left(z^{n} \delta_{\mu}{ }^{\nu}+\bar{z}^{-n} \mathcal{O}_{\mu}{ }^{\nu}\right)
$$

where $z \equiv \exp (\tau+i \sigma)$, and $\tau$ is put to zero (the boundary value) later. Then the correlation function is given by

$$
\begin{aligned}
& \left\langle 0\left|\mathrm{~T}\left(X_{\mu}(z, \bar{z}) X_{\nu}(w, \bar{w})\right)\right| 0\right\rangle \\
& =-\frac{1}{4}\left[\eta_{\mu \nu}\left\{\log \left(1-\frac{\bar{w}}{\bar{z}}\right)+\log \left(1-\frac{z}{w}\right)\right\}+\mathcal{O}_{\mu \nu} \log \left(1-\frac{1}{\bar{z} w}\right)+\mathcal{O}_{\mu \nu} \log (1-\bar{w} z)\right] .
\end{aligned}
$$

This should be evaluated at the boundary $\tau_{z}, \tau_{w} \rightarrow 0$. Extracting the short distance behavior $\left(\delta \equiv \sigma_{z}-\sigma_{w}\right)$, we have the boundary correlators as

$$
\begin{array}{r}
\left\langle\xi_{\mu}(z) \xi_{\nu}(w)\right\rangle_{\text {boundary }}=C_{\mu \nu} \log \delta+\text { finite } \\
\left\langle\xi_{\mu}(z) \partial_{\sigma} \xi_{\nu}(w)\right\rangle_{\text {boundary }}=C_{\mu \nu} \frac{-1}{\delta}+\text { finite } \\
\text { where } \quad C_{\mu \nu} \equiv-\frac{1}{2}\left(\eta_{\mu \nu}+\frac{1}{2} \mathcal{O}_{\mu \nu}+\frac{1}{2} \mathcal{O}_{\nu \mu}\right)
\end{array}
$$

There are $\left(\begin{array}{l}4 \\ 2\end{array}\right)=6$ ways to contract two operators in the vertex $\frac{1}{8} \oint \xi^{\delta} \xi^{\rho} \xi^{\nu}\left(\xi^{\mu}\right)^{\prime} \partial_{\delta} \partial_{\rho} F_{\nu \mu}[\bar{X}]$ appearing in fig. 1. Noting the correspondence

$$
\begin{aligned}
\log \delta: & \lim _{\delta \rightarrow 0} \log \left(1-e^{i \delta}\right)=-\lim _{\delta \rightarrow 0}\left(e^{i \delta}+\frac{1}{2} e^{2 i \delta}+\frac{1}{3} e^{3 i \delta}+\cdots\right)=-\sum_{n \geq 1} \frac{1}{n}=-\zeta(1), \\
\frac{1}{\delta}: & \lim _{\delta \rightarrow 0} \frac{-i e^{i \delta}}{1-e^{i \delta}}=-i \lim _{\delta \rightarrow 0}\left(e^{i \delta}+e^{2 i \delta}+e^{3 i \delta}+\cdots\right)=-i \sum_{n \leq 1} 1=-i \zeta(0),
\end{aligned}
$$


one can see that the contraction (3.22) brings about no divergence after the $\zeta$ function regularization. Therefore we need only to pick up half (three) of the contractions as

$$
\begin{array}{r}
\frac{-i}{8} \oint d \sigma \partial_{\delta} \partial_{\rho} F_{\nu \mu}\left(\xi^{\delta} \xi^{\rho} \xi^{\nu}\left(\xi^{\mu}\right)^{\prime}+\xi^{\delta} \prod^{\rho} \xi^{\nu}\left(\xi^{\mu}\right)^{\prime}+\xi^{\delta} \xi^{\rho} \xi^{\nu}\left(\xi^{\mu}\right)^{\prime}\right) \\
=\frac{i}{4} \log \delta \oint d \sigma \xi^{\mu}\left(\xi^{\nu}\right)^{\prime}\left(\frac{1}{1+F}\right)^{\rho \delta} \partial_{\delta} \partial_{\rho} F_{\mu \nu}
\end{array}
$$

Comparing this result with the propagator in eq. (3.16), the one-loop divergence in the propagator is absorbed into the redefinition of the field strength as

$$
F_{\mu \nu}^{\mathrm{red}}(x) \equiv F_{\mu \nu}(x)+\frac{1}{2} \zeta(1)\left(\frac{1}{1+F}\right)^{\rho \delta} \partial_{\rho} \partial_{\delta} F_{\mu \nu} .
$$

This agrees with eq. (3.12), hence the divergences in the boundary state correspond to the quantum loop divergence in the propagator in the string $\sigma$ model.

The exponent in the boundary state (2.4) consists of the quadratic combination of the oscillators, therefore the boundary state respects the properties of the propagator in the string $\sigma$ model. Here we start from the single $\sigma$ model action (3.15) and expand it, hence it is plausible that the divergences of both the propagator and the vacuum graph can be absorbed into the same redefinition of the gauge field.

\section{Corrections to the D-brane action}

Having defined the boundary state $\left|\mathcal{B}\left(A_{\mu}(x)\right)\right\rangle$ for an arbitrary configuration of the gauge field $A_{\mu}(x)$, our next task in this section is to extract a particular part of the boundary state which describes the interaction with the massless modes of the closed string. This contributes to the corrections to the D-brane action.

As confirmed in ref. [23], the couplings between the gauge field on the D-brane and the closed string field are described in terms of SFT as $\langle\Phi \mid B(F)\rangle$. Thus this interaction (source term) is naturally generalized to the one with non-constant field strength:

$$
\langle\Phi \mid \mathcal{B}\rangle=\left\langle\Phi \mid B\left(F^{\text {red }}(x)\right)\right\rangle+\left\langle\Phi\left|\left[\begin{array}{l}
\text { creation operators } \\
\text { with finite coefficients }
\end{array}\right]\right| B(F(x))\right\rangle .
$$

Here we have substituted the representation (3.11) of $|\mathcal{B}\rangle$, therefore now we adopt the approximation considered in the previous section.

The first term on the RHS of eq. (4.1) gives the same contribution to the part of the D-brane action linear in the closed string massless fields, as the one obtained in the case of 
the constant field strength $\Phi \cdot B(F)$ in ref. [23]. This is because, after putting the coefficient of the $\zeta(1)$ term in eq. (3.13) to zero, then we see $F^{\text {red }}=F$. The result is [23]

$$
\begin{aligned}
\left\langle\Phi \mid B\left(F^{\mathrm{red}}(x)\right)\right\rangle=\frac{-T_{p}}{2} & \int d^{p+1} x(-\operatorname{det}(\eta+F(x)))^{-\zeta(0)} \\
& {\left[\operatorname{tr}(h+b)(1+F(x))^{-1}+\left\{-2+\frac{4}{d-2} \operatorname{tr}(1+F(x))^{-1}\right\} D\right] . }
\end{aligned}
$$

Here $h_{\mu \nu}$ is the fluctuation of the metric field, $b_{\mu \nu}$ is the Kalb-Ramond two-form field, and $D$ is the dilaton field. The divergent $\zeta(0)$ is regularized by the zeta-function regularization. The resultant quantity (4.2) is a part of the well known D-brane action linear in the closed string massless fields, p $^{2}$

$$
-\left.T_{p} \int d^{p+1} x e^{-D} \sqrt{-\operatorname{det}\left(G_{\mu \nu}+b_{\mu \nu}+F_{\mu \nu}^{\mathrm{red}}(x)\right)}\right|_{\text {linear in } D, h_{\mu \nu}, b_{\mu \nu}} .
$$

The metric in this action is the string metric. If which is related to the fluctuation $h$ of the Einstein metric through Weyl rescaling as

$$
G_{\mu \nu}=e^{4 D /(d-2)}\left(\eta_{\mu \nu}+h_{\mu \nu}\right)
$$

The second term in eq. (4.1) gives finite corrections to the D-brane action, that include two derivatives acting on the field strength $F(x)$. In order for the bracket to be expressed in terms of, for example, the massless component fields of the closed string, the oscillator contraction should be performed. Explicitly, the second term on the RHS of eq. (3.11) reads

$$
\begin{aligned}
& {\left[\begin{array}{l}
\text { creation operators } \\
\text { with finite coefficients }
\end{array}\right]|B(F)\rangle} \\
& =-\frac{1}{8} \partial_{\rho} \partial_{\delta} F_{\mu \nu}\left[J^{\kappa \delta} J^{\rho \lambda} J^{\mu \nu}\right] \alpha_{-1 \kappa}^{(-)} \alpha_{-1 \lambda}^{(+)}|B(F)\rangle \\
& \quad+\frac{1}{64} \partial_{\gamma} F_{\alpha \beta} \partial_{\rho} F_{\mu \nu}\left[J^{\rho \gamma} J^{\beta \nu}\left(J^{\mu \alpha}+J^{\alpha \mu}\right)\right]|B(F)\rangle \\
& \quad+\frac{1}{32} \partial_{\gamma} F_{\alpha \beta} \partial_{\rho} F_{\mu \nu}\left[2 J^{\kappa \mu} J^{\nu \beta} J^{\gamma \rho} J^{\alpha \lambda}-J^{\alpha \mu} J^{\nu \beta} J^{\kappa \rho} J^{\gamma \lambda}\right] \alpha_{-1 \kappa}^{(-)} \alpha_{-1 \lambda}^{(+)}|B(F)\rangle \\
& \quad+\cdots
\end{aligned}
$$

8 The terms of higher powers in the closed string fields $h_{\mu \nu}, b_{\mu \nu}$ and $\phi$ which exist in the ordinary D-brane action are expected to be reproduced by performing the integrations over the closed string massive modes. Note that thus we obtain a D-brane action of a form before integrating out the massive modes, i.e. we can read couplings between the open string gauge field strength $F$ and closed string massive modes.

9 The metric field in the D-brane action is defined by an induced one on the D-brane, and we have investigated its dependence in ref. [23] by considering the tilt of the D-brane. We have seen the perfect agreement with the string $\sigma$ model approach, in the case of the constant field strength and the constant tilt angle. Further generalization on general deformation of the D-brane surface will be presented in our next paper 25. 
where the matrix $J^{\mu \nu}$ is defined as

$$
J=1+\mathcal{O}(x)=\frac{2}{1+F(x)}
$$

In (4.5) we have written explicitly only those terms which contain the closed string massless modes, since these terms are necessary for the evaluation of the D-brane action. The omitted part "..." in eq. (4.5) consists of an infinite number of terms of massive excitations, such as $\sum_{l \geq 2} \alpha_{-l}^{(-)} \alpha_{-l}^{(+)}|0\rangle$ or $\left(\alpha^{\dagger}\right)^{n}|0\rangle$ with $n \geq 3$.

Note that the boundary state for the constant field strength $B(F)$ and the massless part of the string field state $\Phi_{\text {massless }}$ have even number of $\alpha$ oscillators. (We ignore the auxiliary fields (denoted as $b_{M}(x)$ and $e_{M}(x)$ in ref. [23]) in $\Phi$, since its bracket in eq. (4.1) vanishes due to the contraction of the ghost oscillators.) Thus contribution of the terms consisting of odd number of $\alpha$ oscillators disappears. With the substitution of eq. (4.5), we find that the second term in eq. (4.1), which is the correction to the D-brane action, equals

$$
\begin{aligned}
& \frac{T_{p}}{64} \sqrt{-\operatorname{det}(\eta+F)}\left(h_{\lambda \kappa}+b_{\lambda \kappa}+\frac{4}{d-2} \eta_{\lambda \kappa} D\right) \\
& \times\left[\partial_{\rho} \partial_{\delta} F_{\mu \nu}\left(J^{\kappa \delta} J^{\rho \lambda} J^{\mu \nu}\right)-\frac{1}{4} \partial_{\gamma} F_{\alpha \beta} \partial_{\rho} F_{\mu \nu}\left(2 J^{\kappa \mu} J^{\nu \beta} J^{\gamma \rho} J^{\alpha \lambda}-J^{\alpha \mu} J^{\nu \beta} J^{\kappa \rho} J^{\gamma \lambda}\right)\right] \\
&-\frac{T_{p}}{256} \sqrt{-\operatorname{det}(\eta+F)}\left[\left(h_{\lambda \kappa}+b_{\lambda \kappa}\right) J^{\kappa \lambda}+\right.\left.\left(-4+\frac{4}{d-2} J_{\lambda}^{\lambda}\right) D\right] \\
& \times \partial_{\gamma} F_{\alpha \beta} \partial_{\rho} F_{\mu \nu}\left[J^{\rho \gamma} J^{\beta \nu}\left(J^{\mu \alpha}+J^{\alpha \mu}\right)\right] .
\end{aligned}
$$

The factor $\zeta(0)$ has been regularized in the same way as before.

\section{Conclusion and discussion}

We have constructed a new boundary state which has the degrees of freedom of an arbitrary configuration of the gauge field $A_{\mu}(x)$ on the D-brane. This state is defined by using the closed SFT gauge transformation acting on a conventional boundary state, inspired from the fact that a gauge transformation of a string $\sigma$ model generates all modes of the gauge field. The new boundary state is BRST invariant and surely satisfies the nonlinear boundary conditions with the non-constant filed strength.

The new boundary state includes divergences of the order $\zeta(1)$ and $\zeta(0)$. The $\zeta(0)$ term can be regularized by the ordinary zeta-function regularization method, whilst the intrinsic divergence of $\zeta(1)$ can be absorbed into the redefinition of the gauge field. This redefinition exactly coincides with the one-loop calculation for the gauge field coupling in a string $\sigma$ model. 
In order for the generalized boundary state to be well-defined, the divergent quantity should vanish, hence this constraint forces the gauge field to satisfy a differential equation. This equation is identical with the conformal invariance condition $\beta(A)=0$ derived in the string $\sigma$ model approach. The origin of this coincidence is studied in the $\sigma$ model side, and we have found that the divergences in the new boundary state can be identified with the one-loop contribution to the worldsheet propagator in the string $\sigma$ model.

Utilizing this boundary state, we have computed the derivative correction terms in the D-brane action, by extracting the part corresponding to the massless excitations of the closed string. After putting the divergence in the redefined gauge field to zero, we have obtained couplings between the derivatives of the field strength $\left(\partial \partial F\right.$ and $\left.(\partial F)^{2}\right)$ and the closed string massless fields $\left(h_{\mu \nu}, b_{\mu \nu}\right.$ and $\left.D\right)$.

It might be possible to apply the procedure given in ref. [23] to obtain a gauge invariant complete SFT action. Although this seems to be straightforward, we find it actually complicated. Only with a naive extension of the method in ref. [23], there may not be corrected Born-Infeld action whose variation under the SFT gauge transformation cancels the surface term stemming from the source term $\langle\Phi \mid \mathcal{B}\rangle$.

There still remains many subjects to be studied. In this paper we have argued the nonconstant mode of the field strength on the D-brane, but it is more intrinsic to introduce massive modes of the open strings. Though the couplings to the massive modes of the closed strings has been manifested in ref. [23], the boundary state contains infinite number of product of the gauge field, which is a sign of the massive open strings. To construct more general state, the SFT gauge transformation will play an important role. The closed SFT adopted in sec. 2 has been generalized to a open-closed mixed system [28], hence some correspondence between our source term and open-closed coupling in ref. [28 may help to understand the boundary state further. At least, the corrections (4.7) may be reproduced in the low energy sector of the system of ref. [28].

One of the other subjects is concerning the supersymmetry. Unfortunately, in the SFT scheme it is hard to discuss the supersymmetry because of some schematic problems [29]. A lot of interesting aspects of D-branes are on the basis of the supersymmetry. We expect that the definition of the new boundary state given in this paper can be generalized to the supersymmetric one. This sort of generalization is seen in ref. 18], in which the case of constant field strength was treated. With use of the generalized supersymmetric boundary state, one can calculate corrections to the D-brane actions in the superstring case as well as various interesting quantities [25]. 


\section{Acknowledgments}

I am indebted to H. Hata for his collaboration at the early stage of this work. I would like to thank also A. Hashimoto, Y. Matsuo, and M. Akashi for useful comments. I am grateful to K. Furuuchi and N. Ishibashi for valuable discussions and careful reading of this manuscript. This work is supported in part by Grant-in-Aid for Scientific Research from Ministry of Education, Science, Sports and Culture (\#3160). I appreciate hospitality of the organizers of Summer Institute ' 99 where a part of this work was argued.

\section{Appendix}

\section{A Calculation of the $*$ product for an arbitrary $\zeta_{\mu}(x)$}

In order to view the meaning of the unitary rotational operator $U[A]$, it is important to check that the definition (2.18) really follows from a gauge rotation (* product) in SFT. What should be checked is

$$
|\mathcal{B}\rangle=|B(F=0)\rangle-2\left|\Lambda\left(-A_{\mu}(x)\right) * B(F=0)\right\rangle
$$

for an infinitesimal $A_{\mu}(x)$ (as a parameter in the $\Lambda$ ), instead of the previous $\zeta_{\mu}(x)$. It is needed only to trace the app. B of ref. [23], for $\zeta_{\mu}(x)$ with arbitrary powers in $x$. We will present only the summary of the calculation. In this case we should take into consideration the $F_{p^{2}}$ term (eq. (B.6) in 23])

$$
\exp F_{p^{2}}=\exp \left(-\frac{1}{4} \eta^{\mu \nu} p_{\mu} p_{\nu} \log \left|\frac{e \alpha_{2}}{\epsilon}\right|+\cdots\right)
$$

in the three string vertex operator $V_{123}$. This $F_{p^{2}}$ factor is quadratic in $p$, thus when treating $\zeta_{\mu}$ linear in $x\left(=i \frac{\partial}{\partial p}\right)$ as in 23 its contribution disappears. But for higher powers in $x$, the logarithmic divergence $\log \left|\frac{\epsilon}{\alpha}\right|$ comes out. This divergences are absorbed into the normal ordering divergences of the $X$ 's in $\zeta_{\mu}(X)$, but its finite part remains ambiguous. For example, the gauge transformation parameter

$$
\zeta_{\mu}(x)=\zeta_{\mu \nu \rho} x^{\nu} x^{\rho}+\zeta_{\mu \nu \rho \delta} x^{\nu} x^{\rho} x^{\delta}
$$


results in the identity

$$
-2|\Lambda * B(F)\rangle=\left[\frac{i}{\pi} \oint \partial_{\sigma} X^{\mu} \zeta_{\mu}(X)+c \cdot \frac{i}{\pi} \oint \partial_{\sigma} X^{\mu}\left(\zeta_{\mu \nu \rho}{ }^{\rho}+\zeta_{\mu \rho \nu}{ }^{\rho}+\zeta_{\mu \rho}{ }^{\rho}{ }_{\nu}\right) X^{\nu}\right]|B(F)\rangle,
$$

where $c$ is a finite unknown constant (contractions of $\zeta_{\mu \nu \rho \delta}$ comes from the flat metric $\eta^{\mu \nu}$ in eq. (A.2)).

In this paper we assume that $c=0$, since if we define $|\mathcal{B}\rangle$ with non-zero $c$ using the above eq. (A.4), the generalized nonlinear boundary condition (2.23) does not follow. After all, together with the above results and the definitions (2.15) (2.18), the gauge transformation of the dynamical variable $A_{\mu}(x)$ of the new boundary state $|\mathcal{B}\rangle$ generated by the SFT $*$ product is (2.16), for an arbitrary number of powers in $x_{\mu}$.

\section{B Calculation of the divergence in the boundary state}

In this appendix we present the calculation to obtain eq. (3.11), changing all the oscillators into creation operators using the boundary condition (3.8). We show here the calculation of only the last term in eq. (3.5), since this term is the most bothersome and other terms can be evaluated in the same way. Considering the corresponding mode of the gauge field

$$
A_{\mu}(x)=\zeta_{\mu \nu \rho} x^{\nu} x^{\rho}, \quad \text { with } \zeta_{\mu \nu \rho}=\zeta_{\mu \rho \nu}
$$

the last term in eq. (3.5) is written as

$$
\begin{aligned}
T \equiv \frac{-1}{32} \zeta_{\mu \nu \rho} \zeta_{\alpha \beta \gamma}\left[\sum_{n, m} \frac{1}{n m}(\right. & \left.\left.\alpha_{-n-m}^{(+) \mu}-\alpha_{n+m}^{(-) \mu}\right)\left(\alpha_{n}^{(+) \nu}-\alpha_{-n}^{(-) \nu}\right)\left(\alpha_{m}^{(+) \rho}-\alpha_{-m}^{(-) \rho}\right)\right] \\
& \times\left[\sum_{k, l} \frac{1}{k l}\left(\alpha_{-k-l}^{(+) \alpha}-\alpha_{k+l}^{(-) \alpha}\right)\left(\alpha_{k}^{(+) \beta}-\alpha_{-k}^{(-) \beta}\right)\left(\alpha_{l}^{(+) \gamma}-\alpha_{-l}^{(-) \gamma}\right)\right] .
\end{aligned}
$$

This is evaluated on the boundary state $|B(F(x))\rangle$. After changing all the oscillators into creation operators, then we are led to the following form symbolically:

$$
T|B(F(x))\rangle=\left[\left(\alpha^{\dagger}\right)^{6}+\left(\alpha^{\dagger}\right)^{4}+\left(\alpha^{\dagger}\right)^{2}+c\right]|B(F(x))\rangle .
$$

On the RHS, the first two terms have finite coefficients and furthermore are not relevant for the massless modes in the closed string excitations, hence it is not necessary to evaluate them explicitly. The third term quadratic in $\alpha$ and the last constant term $c$ contain divergences and 
also finite contributions. The $\left(\alpha^{\dagger}\right)^{2}$ term is given as

$$
\begin{aligned}
& \left(\alpha^{\dagger}\right)^{2}=\sum_{m+n>0, m<0} \alpha_{-n-m}^{(-) \sigma} \alpha_{-n-m}^{(+) \delta}\left[\frac{2}{m(n+m)}\left(-J^{\sigma \mu} J^{\nu \alpha} J^{\beta \rho} J^{\gamma \delta}-J^{\mu \delta} J^{\alpha \nu} J^{\rho \beta} J^{\sigma \gamma}\right)\right. \\
& +\frac{2}{n(n+m)}\left(-J^{\sigma \mu} J^{\nu \beta} J^{\alpha \rho} J^{\gamma \delta}-J^{\mu \delta} J^{\beta \nu} J^{\rho \alpha} J^{\sigma \gamma}\right) \\
& \left.+\frac{-2}{m n}\left(-J^{\sigma \mu} J^{\nu \beta} J^{\gamma \rho} J^{\alpha \delta}-J^{\mu \delta} J^{\beta \nu} J^{\rho \gamma} J^{\sigma \alpha}\right)\right] \\
& +\sum_{m>0, n>0} \alpha_{-n-m}^{(-) \sigma} \alpha_{-n-m}^{(+) \delta}\left[\frac{-2}{m(n+m)}\left(-J^{\sigma \mu} J^{\nu \alpha} J^{\rho \beta} J^{\gamma \delta}-J^{\mu \delta} J^{\alpha \nu} J^{\beta \rho} J^{\sigma \gamma}\right)\right. \\
& +\frac{-2}{n(n+m)}\left(-J^{\sigma \mu} J^{\nu \beta} J^{\rho \alpha} J^{\gamma \delta}-J^{\mu \delta} J^{\beta \nu} J^{\alpha \rho} J^{\sigma \gamma}\right) \\
& \left.+\frac{2}{m n}\left(-J^{\sigma \mu} J^{\nu \beta} J^{\rho \gamma} J^{\alpha \delta}-J^{\mu \delta} J^{\beta \nu} J^{\gamma \rho} J^{\sigma \alpha}\right)\right] \\
& + \text { other summations. }
\end{aligned}
$$

Here "other summations" denotes the summation in the other four regions in the $(m, n)$-plane divided by three lines: $m=0, n=0, m+n=0$. The other summation is performed in the same manner with slight changes of the indices. From this expression we can extract the divergences $\zeta(1)$. For example, from the first summation we have

$$
\begin{aligned}
\zeta(1) \sum_{\widetilde{n}>0} \frac{2}{\widetilde{n}} \alpha_{-\widetilde{n}}^{(-) \sigma} \alpha_{-\widetilde{n}}^{(+) \delta}[- & \left.-J^{\sigma \mu} J^{\nu \alpha} J^{\beta \rho} J^{\gamma \delta}-J^{\mu \delta} J^{\alpha \nu} J^{\rho \beta} J^{\sigma \gamma}\right) \\
& \left.+\left(-J^{\sigma \mu} J^{\nu \beta} J^{\alpha \rho} J^{\gamma \delta}-J^{\mu \delta} J^{\beta \nu} J^{\rho \alpha} J^{\sigma \gamma}\right)\right]
\end{aligned}
$$

where we have defined $\widetilde{n} \equiv n+m$. This is because, in this case we should sum over $\widetilde{n}$ as the index of the oscillators, and for a fixed $\widetilde{n}$, divergent quantities proportional to $\zeta(1)$ stem from the first and the second terms in the first summation in eq. (B.4) (the factor of the third term $-2 / n m$ does not give the divergence). In the same way, one finds that the second summation in eq. (B.4) does not bring about the divergence. Collecting all the divergent terms, we can verify the following equality:

$$
\text { divergent terms of }\left(\alpha^{\dagger}\right)^{2}=\left|B_{\mathrm{N}}(F+\delta F)\right\rangle-\left|B_{\mathrm{N}}(F)\right\rangle
$$

with

$$
\delta F^{\lambda \kappa} \equiv-\zeta(1) \cdot \frac{1}{2} \zeta_{\mu \nu \rho} \zeta_{\alpha \beta \gamma}\left[J^{\alpha \mu} J^{\nu \beta} \eta^{\rho \lambda} \eta^{\gamma \kappa}+J^{\mu \beta} J^{\gamma \nu} \eta^{\alpha \kappa} \eta^{\rho \lambda}-J^{\nu \beta} J^{\gamma \mu} \eta^{\alpha \kappa} \eta^{\rho \lambda}\right]-(\kappa \leftrightarrow \lambda) .
$$

In the same way, the constant term $c$ in eq. $($ B.3 $)$ is evaluated, and the result is

$$
\text { divergent terms of } c=N(F+\delta F)-N(F)
$$


with the above same $\delta F($ B.7). These give the relation (3.11), using the identification

$$
\partial_{\gamma} F_{\alpha \beta}=2\left(\zeta_{\alpha \beta \gamma}-\zeta_{\beta \alpha \gamma}\right) .
$$

\section{References}

[1] J. Dai, R. G. Leigh, and J. Polchinski, Mod. Phys. Lett. A4 (1989) 2073 ;

R. G. Leigh, Mod. Phys. Lett. A4 (1989) 2767.

[2] C. M. Hull and P. K. Townsend, Nucl. Phys. B438 (1995) 109.

[3] R. G. Leigh, Mod. Phys. Lett. A4 (1989) 2767 ;

M. Aganagic, C. Popescu and J. H. Schwarz, Phys. Lett. B393 (1997) 311.

[4] C. Schmidhuber, Nucl. Phys. B467 (1996) 146.

[5] S. P. de Alwis and K. Sato, Phys. Rev. D53 (1996) 7187 hep-th/9601167 ;

A. A. Tseytlin, Nucl. Phys. B469 (1996) 51, hep-th/9602064;

Y. Lozano, Phys. Lett. B399 (1997) 233, hep-th/9701186 ;

M. Aganagic, J. Park, C. Popescu and J. H. Schwarz, Nucl. Phys. B496 (1997) 215, hep-th/9702133;

Y. Igarashi, K. Itoh, K. Kamimura and R. Kuriki, JHEP 9803 (1998) 002, hep-th/9801118;

I. Oda, Phys. Lett. B430 (1998) 242, hep-th/9802152 ;

Y. Igarashi, K. Itohand K. Kamimura, Nucl. Phys. B536 (1998) 469, hep-th/9806161.

[6] H. Ishikawa, Y. Matsuo, Y. Sugawara and K. Sugiyama, Phys. Lett. B388 (1996) 292.

[7] H. Dorn and H. -J. Otto, Z. Phys. C32 (1986) 599.

[8] A. Abouselsaood, C. G. Callan, C. R. Nappi and S. A. Yost, Nucl. Phys. B280(FS18) (1987) 599.

[9] C. G. Callan, C. Lovelace, C. R. Nappi and S. A. Yost, Nucl. Phys. B288 (1987) 525.

[10] E. Bergshoeff, E. Sezgin, C. N. Pope and P. K. Townsend, Phys. Lett. B188 (1987) 70.

[11] E. S. Fradkin and A. A. Tseytlin, Phys. Lett. 163B (1985) 123 ;

R. R. Metsaev, M. A. Rakhmanov and A. A. Tseytlin, Phys. Lett. 193B (1987) 207. 
[12] M. R. Garousi, JHEP 9812 (1998) 008, hep-th/9805078 ;

M. R. Garousi, R. C. Myers Nucl. Phys. B542 (1999) 73, hep-th/9809100.

[13] O. D. Andreef and A. A. Tseytlin, Nucl. Phys. B311 (1988/89) 205 ; Mod. Phys. Lett. A3 (1988) 1349 ;

Y. Kitazawa, Nucl. Phys. B289 (1987) 599.

[14] J. Bagger and A. Galperin, Phys. Lett. B412 (1997) 296, hep-th/9707061.

[15] N. Kaloper and K. A. Meissner, Phys. Rev. D56 (1997) 7940, hep-th/9705193.

[16] C. P. Bachas, P. Bain and M. B. Green, JHEP 9905 (1999) 011, hep-th/9903210.

[17] C. G. Callan, C. Lovelace, C. R. Nappi and S. A. Yost, Nucl. Phys. B293 (1987) 83.

[18] C. G. Callan, C. Lovelace, C. R. Nappi and S. A. Yost, Nucl. Phys. B308 (1988) 221.

[19] C. G. Callan, C. Lovelace, C. R. Nappi and S. A. Yost, Phys. Lett. B206 (1988) 41.

[20] J. Polchinski and Y. Cai, Nucl. Phys. B296 (1988) 91.

[21] N. Ishibashi, Mod. Phys. Lett. A4 (1989) 251.

[22] M. B. Green and M. Gutperle, Nucl. Phys. B476 (1996) 484, hep-th/9604091;

P. Di Vecchia, M. Frau, A. Lerda and A. Liccardo, hep-th/9906214.

[23] K. Hashimoto and H. Hata, Phys. Rev. D56 (1997) 5179, hep-th/9704125.

[24] H. Hata, K. Itoh, T. Kugo, H. Kunitomo and K. Ogawa, Phys. Rev. D35 (1987) 1318.

[25] K. Hashimoto, to appear.

[26] C. G. Callan and I. R. Klebanov, Nucl. Phys. B465 (1996) 473, hep-th/9511173.

[27] M. Billo, D. Cangemi and P. Di Vecchia, Phys. Lett. B400 (1997) 63, hep-th/9701190 ;

H. Arfaei and M. M. Sheikh-Jabbari, Nucl. Phys. B526 (1998) 278, hep-th/9709054.

[28] T. Kugo and T. Takahashi, Prog. Theor. Phys. 99 (1998) 649, hep-th/9711100;

T. Asakawa, T. Kugo and T. Takahashi, Prog. Theor. Phys. 100 (1998) 831, hep-th/ 9807066 ; hep-th/9905043.

[29] N. Berkovits, Phys. Lett. B388 (1996) 743, hep-th/9607070, and references therein. 